\title{
Comportamiento histerético del acero de refuerzo longitudinal en elementos de borde de muros estructurales de hormigón armado
}

\author{
Jessica Sarango $^{\mathrm{a}}$, Ana G. Haro ${ }^{\mathrm{b}}$ \\ ${ }^{a}$ Estudiante, Carrera de Ingeniería Civil, Universidad de las Fuerzas Armadas ESPE, Sangolquí, Ecuador \\ ${ }^{\text {b }}$ Profesora, Departamento de Ciencias de la Tierra y la Construcción, Universidad de las Fuerzas Armadas ESPE, Sangolquí, \\ Ecuador \\ jfsarango@espe.edu.ec, agharo@espe.edu.ec
}

\begin{abstract}
Resumen-El pandeo inelástico fuera de plano en muros estructurales de hormigón armado es un mecanismo de falla que había sido observado únicamente en laboratorios hasta antes de los sismos de Chile (2010) y Nueva Zelanda (2011). Solicitaciones reversibles en el plano de resistencia concentran el daño en los elementos de borde a lo largo de la zona plástica. Es en esta región donde el comportamiento histerético del refuerzo longitudinal juega un rol fundamental en el mecanismo en mención. Estudios previos han demostrado que, al limitar la deformación por tensión a un valor máximo en función del contenido de refuerzo, las propiedades mecánicas y geométricas de la zona de compresión, se podría garantizar un desempeño estructural estable. En base a estos estudios donde se ensayaron elementos de borde ante cargas axiales cíclicas, en la presente investigación se realiza una comparación de resultados para las componentes que caracterizan al comportamiento histerético del refuerzo principal con aquellos arrojados de un modelamiento con elementos finitos. Del análisis se observa que los resultados del modelo computacional son similares a aquellos de la fase experimental del proyecto base, constituyéndose en una herramienta eficiente para el estudio del pandeo fuera de plano en muros estructurales.
\end{abstract}

Palabras Claves-Comportamiento histerético del refuerzo, pandeo inelástico, elementos de borde de muros estructurales de HA.

\begin{abstract}
The out-of-plane buckling instability in reinforced concrete structural walls is a failure mechanism that had only been observed in laboratories until the Chile (2010) and New Zealand (2011) earthquakes. Load reversals applied in the resistance plane concentrate the damage in boundary elements along the plastic zone. It is in this region where the hysteretic behavior of the longitudinal reinforcement plays a primary role within the mentioned mechanism. Previous studies have demonstrated that if tensile strains are limited to a maximum value as a function of steel ratio, the mechanical and geometric properties of the compression zone, a stable structural performance could be guaranteed. Based on these studies where boundary elements were subjected to axial cyclic loading, a comparison of results is made in this research for the components that characterize the hysteretic behavior of the main reinforcement with those determined from a finite element modeling. From the analysis it is observed that the results of the computational model are similar to those of the experimental phase of the base project, constituting an efficient tool for the study of out-of-plane buckling in structural walls.
\end{abstract}

Keywords-Reinforcement hysteretic behavior, inelastic buckling, boundary element of RC structural walls.

\section{INTRODUCCIÓN}

El 22 de febrero del 2010, Chile sufrió las consecuencias catastróficas de un evento sísmico, de magnitud 8.8 y una aceleración de suelo de $0.6 \mathrm{~g}$, que duró aproximadamente tres minutos, afectando a más de 300,000 estructuras [1]. Se observó un fenómeno repetido en edificios de varios pisos que contaban con un sistema de muros estructurales de hormigón armado (MEHA), mismo que únicamente había sido capturado y estudiado en laboratorio. Este modo de falla fue identificado como pandeo fuera de plano, que se desarrolla en los ciclos inelásticos ante solicitaciones en el plano, concentrando el mayor daño en los elementos de borde [2]-[7].

El pandeo fuera de plano fue estudiado por primera vez por Goodsir en 1985 [2], quien observó que las varillas longitudinales servían como única fuente de estabilidad en la región de compresión, tras el desarrollo de fisuras amplias, producto de grandes deformaciones unitarias inelásticas de tensión a lo largo de la zona plástica [3]. Esta investigación sirvió de base para los modelos fenomenológicos creados por Paulay \& Priestley (1993) [4] y Chai \& Elayer (1999) [5] que asociaron los efectos de las cargas en el plano con las deformaciones fuera del plano en MEHA. Estos modelos consideran un criterio de estabilidad en función de las propiedades mecánicas del material y la cuantía de refuerzo en la zona de compresión, sin embargo, las predicciones resultarían conservadoras de acuerdo con [2], [5], [7], [8].

Más tarde, Herrick \& Kowalsky (2016), Haro, Kowalsky \& Chai (2017) y Rosso-Almeida-Beyer (2018) propusieron modelos en base a [4] y [5], limitando las deformaciones máximas unitarias a fin de garantizar la estabilidad en MEHA. Debido a que el refuerzo longitudinal en los elementos de borde sería el único que resiste las cargas de compresión durante la etapa previa al cierre de las fisuras, el análisis de su comportamiento histerético es esencial, y es por ello que en esta investigación se comparan las diferentes componentes de deformación, fundamentándose en los modelos propuestos por Chai \& Elayer (C\&E) [5] y Haro, Kowalsky \& Chai (HKC) [2], que por su forma representarían una alternativa fácil de implementar en procesos de diseño y evaluación.

\section{MÉTODO}

El modelo planteado para el análisis del comportamiento histerético del acero es el de C\&E [5] que captura el pandeo inelástico en elementos de borde (EB) de MEHA expuestos a ciclos de compresión y tracción axial. Esta propuesta 
plantea que la inestabilidad se produce antes de que el muro alcance su capacidad en el plano y que la amplitud de las grietas desarrolladas en una etapa posterior a la cedencia se cierre. En esta fase las deformaciones unitarias por tensión en el refuerzo longitudinal son un parámetro crítico que gobierna la estabilidad fuera del plano.

Es así que este fenómeno puede ser controlado al limitar las deformaciones unitarias por tensión $\left(\varepsilon_{s}\right)$, y para ello es necesario determinar la magnitud máxima previa al aplastamiento del concreto bajo esfuerzos de compresión en la zona de pandeo donde se produce la máxima deflexión fuera de plano.

La deformación unitaria máxima $\left(\varepsilon_{s m}\right)$ se estima utilizando la repuesta idealizada presentada en la Fig. 1, en donde se distinguen tres componentes de deformación que describen el comportamiento histérico del acero longitudinal, y a partir de ello se plantea la ecuación (1) [5].

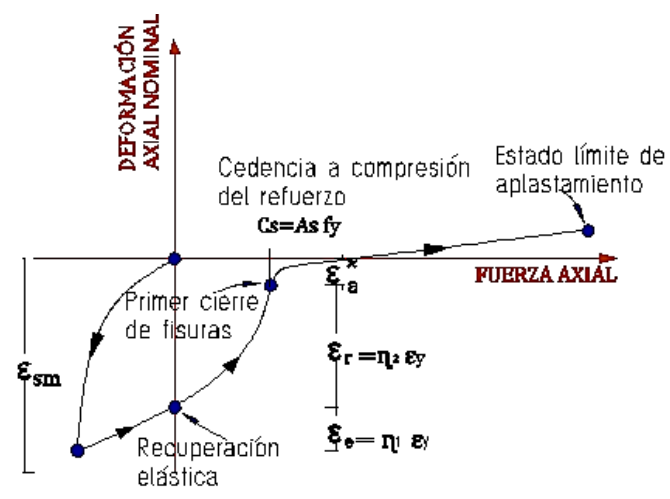

Fig. 1. Estimación de $\varepsilon_{s m}$ en un prisma de H.A. Deformación nominal axial vs fuerza axial [5]

donde $\varepsilon_{a}^{*}$ es la componente que corresponde a la deformación axial en el primer cierre de fisuras, $\varepsilon_{e}$ es la deformación elástica de recuperación por una excursión en tensión y $\varepsilon_{r}$ es la deformación en la recarga asociada a la cedencia del refuerzo longitudinal en compresión.

$$
\varepsilon_{s m}=\varepsilon_{a}^{*}+\varepsilon_{e}+\varepsilon_{r}
$$

$\varepsilon_{e}$ depende de la conexión de transferencia entre el acero y el concreto, principalmente de la contracción del acero, es por ello que esta componente al igual que $\varepsilon_{r}$ puede ser expresada en términos de la cedencia del acero $\varepsilon_{y}$, así: $\varepsilon_{e}=\eta_{1} \cdot \varepsilon_{y}$ y $\varepsilon_{r}=\eta_{2} \cdot \varepsilon_{y}$. Para los coeficientes $\eta_{1}, \eta_{2}$ se recomiendan valores de 1 y 2 , respectivamente [5], con fines de diseño.

Para la estimación de la deformación en el primer cierre de ancho de grietas, se considera una distribución sinusoidal de la curvatura transversal a lo largo de la zona de pandeo. Además, se adopta el criterio de estabilidad planteado por Paulay $\&$ Priestley

$\xi_{c}=0.5 \cdot\left(1+2.35 m-\sqrt{5.53 m^{2}+4.7 m}\right)$, donde $m$ es la relación de refuerzo mecánico $\rho f_{y} / f_{c}^{\prime}, \rho$ es la cuantía de acero y $f_{y}, f^{\prime}{ }_{c}$ son los esfuerzos de cedencia del acero y de compresión del concreto, respectivamente [4]. Es así que $\varepsilon_{a}^{*}=\frac{\pi^{2}}{2}\left(\frac{b_{w}}{L_{o}}\right)^{2} \cdot \xi_{c}$, donde $b_{w}$ corresponde al espesor del muro y $L_{o}$ a la longitud de pandeo que se considera igual a la altura de entrepiso sobre la raíz de tres $h_{s} / \sqrt{3}$ de acuerdo con [2].

Así (2) se replantea al tomar en cuenta los parámetros ya mencionados:

$$
\varepsilon_{s m}=\frac{\pi^{2}}{2}\left(\frac{b_{w}}{L_{o}}\right)^{2} \cdot \xi_{c}+\eta_{1} \cdot \varepsilon_{y}+\eta_{2} \cdot \varepsilon_{y}
$$

Mediante (2), se determina la deformación unitaria máxima por tensión de un EB mostrado en la Fig. 2 cuya geometría, disposición de armado y propiedades mecánicas de los materiales se tomó en función del prisma 2 (P2) de la fase experimental más reciente ensayada en EB con dos capas de refuerzo [2].

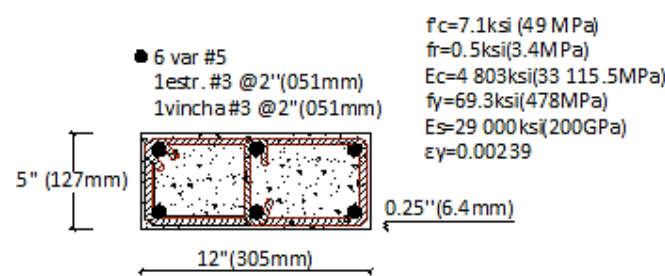

Fig. 2. Sección trasversal del EB a analizar.

Al utilizar lo detallado en la Fig. 2, se llevó a cabo la modelación computacional de P2 en el software SeismoStruct, un paquete de elementos finitos (EF) capaz de predecir el comportamiento de estructuras bajo cargas dinámicas y estáticas así como su desempeño tanto en el rango elástico como inelástico, que utiliza una distribución optimizada de Gauss-Lobatto como método de integración numérica, el mismo que comprende al menos un punto de control en cada extremo de un miembro [6]. Mediante este programa se condujo un análisis no lineal Time-history estático con elementos basados en fuerzas que ha demostrado ser una formulación más confiable, ya que, siempre proporciona una solución exacta para los elementos tipo prismáticos debido a que es independiente del comportamiento constitutivo del material [7], [8]. Para hacer más preciso este modelo se utiliza EF, para representar a un EB con apoyos fijos en ambos extremos. Como se espera que se desempeñe en el rango inelástico, se considera 200 fibras de sección y 6 secciones de integración.

En cuanto a los materiales, el modelo utilizado para el concreto es el de Mander (con_man), mientras que, para el acero se considera el modelo de Menegotto \& Pinto (stl_mp), que se ajustan más al comportamiento inelástico de análisis. Los parámetros de ajuste considerados para definir el refuerzo longitudinal y transversal se obtienen de la experimentación y calibración llevadas a cabo por [3], como se muestra en la Tabla I.

TABLA I

PARÁmetros MecÁnicos de LA ARMADURA PARA EL MODELAdO EN SEISMOSTRUCT [3]

\begin{tabular}{lccccc}
\hline \hline Parámetro & $\boldsymbol{a}_{\mathbf{1}}$ & $\boldsymbol{a}_{\mathbf{2}}$ & $\boldsymbol{a}_{\mathbf{3}}$ & $\boldsymbol{a}_{\mathbf{4}}$ & $\boldsymbol{R}_{\boldsymbol{o}}$ \\
\hline Valor & 2.0 & 18 & 0.15 & 0.025 & 2.0 \\
\hline \hline
\end{tabular}

El parámetro $R_{o}$ controla la forma de transición de la curva entre la rigidez inicial y la post cedencia, esto toma en 
cuenta el efecto Bauschinger y los picos de los bucles de las curvas de histéresis. Los coeficientes $a_{1}, a_{2}$ gradúan los cambios de $R_{o}$ para establecer el parámetro de curvatura actualizado $R_{n}$. Los coeficientes $a_{3}, a_{4}$ definen el grado en que se incluye el endurecimiento isotrópico en la respuesta del material de tensión-deformación [6].

En cuanto a la parte experimental, el comportamiento histerético de las caras sur y norte de P2 sujeto a cargas cíclicas axiales se presenta en la Fig. 3 y Fig. 4, respectivamente. En estas figuras se visualiza diferentes etapas de la respuesta axial del prisma, capturadas durante el último ciclo donde se produjo inestabilidad de pandeo fuera de plano, misma que se ilustra en la Fig. 5 con la identificación respectiva de las caras del prisma.

El modelo HKC se fundamentó en el de Chai \& Elayer [5] con la diferenciación en la distribución de la curvatura transversal a lo largo de la zona de pandeo considerada como polinomial de segundo grado, adicionalmente se toma en cuenta el efecto de la cuantía de acero en las components $\varepsilon_{e}$ y $\varepsilon_{r}$. Este modelo fue calibrado y validado a través de los resultados de estudios experimentales y analíticos que conformaron parte de dicha investigación [2], y está representado por (3), donde $\rho_{\text {lbe }}$ corresponde a la cuantía de armado longitudinal y el resto de parámetros son los ya mencionados previamente.

$$
\varepsilon_{s m} \leq \frac{8}{3}\left(\frac{b_{w}}{L_{o}}\right)^{2} \cdot \xi_{c}+\left[-2.4+160\left(\frac{b_{w}}{L_{o}}\right)^{2}-1.9 \ln \left(\rho_{l b e}\right)\right] \cdot \varepsilon_{y}
$$

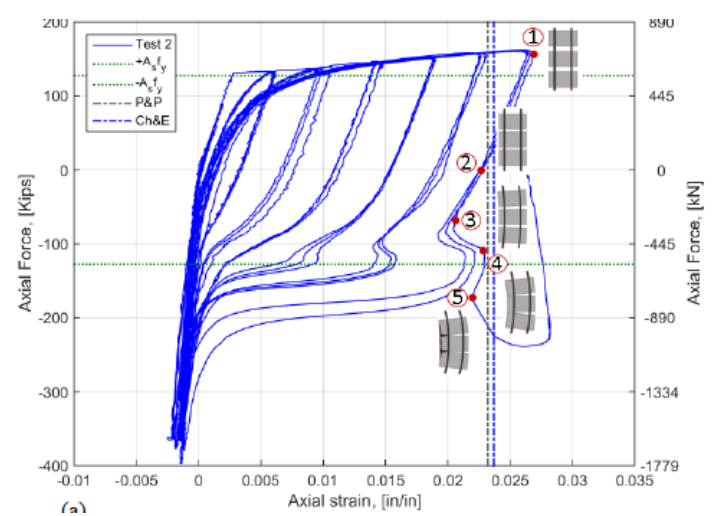

Fig. 3. Fases de la respuesta axial del P2 cara sur [2].

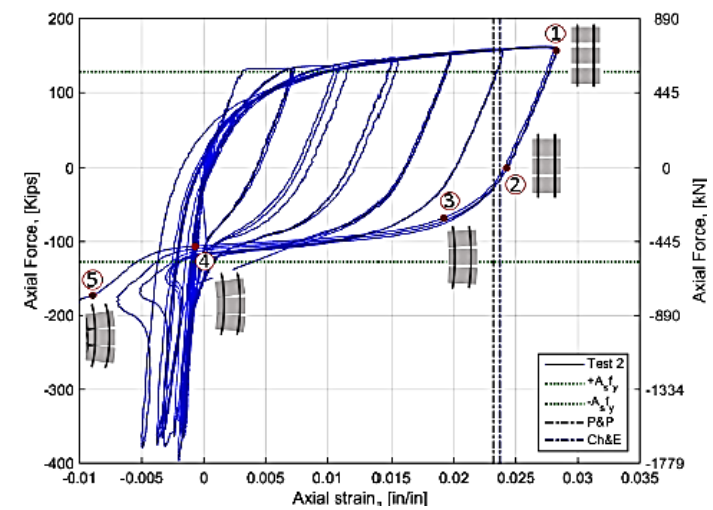

Fig. 4. Fases de la respuesta axial del P2 cara norte [2].

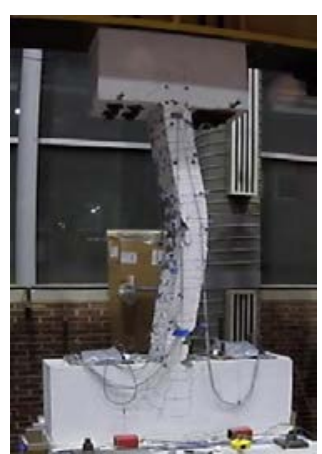

(a)

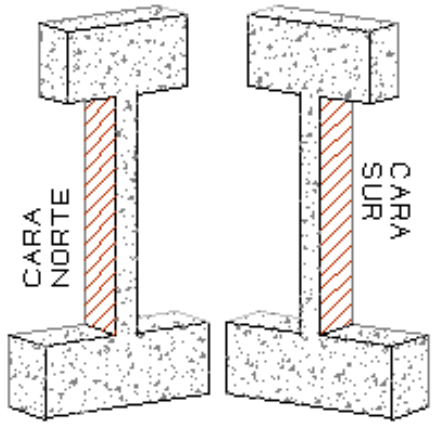

(b)
Fig. 5. (a) Fase final de la experimentación del P2 [2] (b) Ubicación de las caras del prisma.

\section{ANÁLISIS DE RESUltAdOS}

La estimación de la deformación máxima unitaria por tensión y sus componentes obtenida mediante el método planteado por Chai \& Elayer (C\&E) y por el método HKC se presenta en la Tabla II.

TABLA I

COMPONENTES DE LA DEFORMACIÓN MÁXIMA, $\varepsilon_{s m}$

\begin{tabular}{ccccc}
\hline \hline Método & $\boldsymbol{\varepsilon}_{\text {sm }}$ & $\boldsymbol{\varepsilon}_{\boldsymbol{a}}^{*}$ & $\boldsymbol{\varepsilon}_{\boldsymbol{e}}$ & $\boldsymbol{\varepsilon}_{\boldsymbol{r}}$ \\
\hline $\boldsymbol{C} \boldsymbol{E} \boldsymbol{E}$ & 0.02374 & 0.01657 & 0.00239 & 0.00478 \\
$\boldsymbol{H K C}$ & 0.02704 & 0.00896 & \multicolumn{2}{c}{0.01809} \\
\hline \hline
\end{tabular}

El resultado obtenido de la modelación en SeismoStruct se visualiza en la curva de histéresis presentada en la Fig. 6, en la cual $\varepsilon_{s m}=0.02776$ corresponde a la cara convexa y $\varepsilon_{s m}=0.02748$ a la cara cóncava, dónde la falla por pandeo se produjo en el tercer ciclo correspondiente a $12 \varepsilon_{y}$.

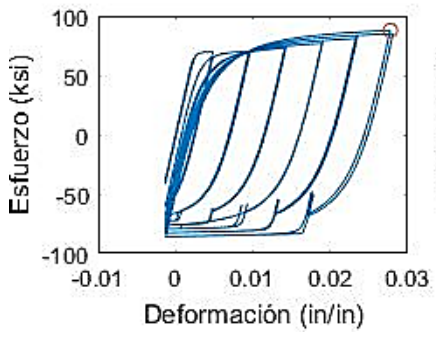

(a)

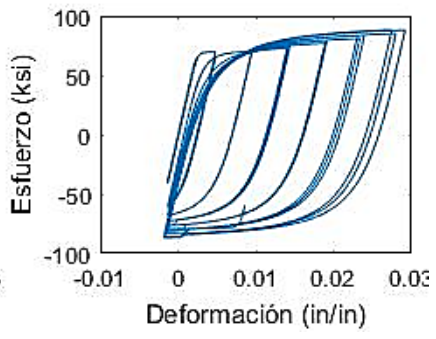

(b)
Fig. 6. Curva de histéresis obtenida de SeismoStruct (a) Cara convexa (b) Cara cóncava.

En cuanto a la experimentación de P2 los valores obtenidos de las curvas de histéresis presentadas en las Fig. 3 y Fig. 4, se sintetiza en la Tabla III.

TABLA II

COMPONENTES EXPERIMENTALES DE $\boldsymbol{\varepsilon}_{\boldsymbol{s m}}$. ADAPTACIÓN [3].

\begin{tabular}{ccccc}
\hline \hline Cara & $\boldsymbol{\varepsilon}_{\boldsymbol{s m}}$ & $\boldsymbol{\varepsilon}_{\boldsymbol{e}}$ & $\boldsymbol{\varepsilon}_{\boldsymbol{a}}^{*}$ & $\boldsymbol{\varepsilon}_{\boldsymbol{r}}$ \\
\hline Sur & 0.02699 & 0.00411 & 0.00902 & 0.01386 \\
Norte & 0.02835 & 0.00416 & 0.01033 & 0.01386 \\
Prom. & $\mathbf{0 . 0 2 7 6 7}$ & $\mathbf{0 . 0 0 4 1 4}$ & $\mathbf{0 . 0 0 9 6 8}$ & $\mathbf{0 . 0 1 3 8 6}$ \\
\hline \hline
\end{tabular}

En cuanto al valor de $\varepsilon_{s m}$ y sus componentes la Tabla IV muestra una comparación entre las distintas formas de 
estimación y la variación en base a la experimentación. El análisis refleja que las predicciones del modelo HKC se aproximan más a los resultados experimentales.

TABLA III

COMPARACIÓN DE RESULTADOS

\begin{tabular}{|c|c|c|c|c|c|c|}
\hline Método & $\bar{\varepsilon}_{s m}$ & $\varepsilon_{a}^{*}$ & $\varepsilon_{e}+\varepsilon_{r}$ & \multicolumn{3}{|c|}{ Variación (\%) } \\
\hline P2 & 0.02767 & 0.00968 & 0.01800 & $\varepsilon_{s m}$ & $\varepsilon_{a}^{*}$ & $\varepsilon_{e}+\varepsilon_{r}$ \\
\hline$C \& E$ & 0.02374 & 0.01657 & 0.00717 & 14.2 & 71.3 & 60.2 \\
\hline HKC & 0.02704 & 0.00896 & 0.01809 & 2.3 & 7.4 & 0.5 \\
\hline SS & 0.02762 & 0.00896 & 0.01866 & 0.2 & 7.4 & 3.7 \\
\hline
\end{tabular}

El modo de falla por pandeo fuera de plano se produjo durante el tercer ciclo de $12 \varepsilon_{y}$, tanto en la fase experimental, como en el modelo computacional que reportó una deformación unitaria máxima promedio de $\varepsilon_{s m}=0.02762$, con un error de $0.2 \%$. El modelo HKC fue capaz de predecir la máxima respuesta con un error de $2.3 \%$. El modelo C\&E resultó conservador reportando un error de 14.2\%.

En lo referente a las componentes de la deformación unitaria máxima, se observa que el modelo de C\&E estima valores en extremo alejados de la respuesta experimental, mientras que las componentes de los modelos HKC y computacional, reportan como máximo un porcentaje de diferencia en orden del 7\%.

\section{CONCLUSIONES}

Se ha realizado un análisis de la respuesta ante acciones cíclicas axiales de prismas de hormigón armado que simulan elementos de bordes de muros estructurales. Al comparar las deformaciones de tensión máximas capturadas previo a la falla por pandeo fuera de plano se concluye que tanto el modelo HKC y el modelo computacional se asemejan a los resultados observados en la fase experimental de [3]. El presente estudio refleja que SeismoStruct constituye una herramienta eficiente para simular acciones sísmicas en elementos de borde de MEHA y capturar el efecto de los parámetros críticos que influyen en el mecanismo de falla asociado al pandeo fuera de plano inelástico.

\section{RECONOCIMIENTOS}

Este trabajo es parte del Proyecto de Investigación "Efecto del espesor de muros estructurales de HA en el modo de falla de pandeo fuera de plano", que se ejecuta en el Departamento de Ciencias de la Tierra y la Construcción de la Universidad de las Fuerzas Armadas ESPE.

\section{REFERENCIAS}

[1] A. Briseño y N. Carreras, “Análisis y diseño de muros estructurales de concreto, considerando las experiencias de los terremotos de Chile 2010 y Nueva Zelanda 2011”, Santiago de Chile: Universidad Católica Andrés Bello, 2013.

[2] A. Haro, "Assessment of the Out-of-Plane Buckling Instability of Ductile Reinforced Concrete Structural Walls”, Raleigh: North Carolina State University. Doctoral thesis, 2017.

[3] W. Goodsir, "The design of coupled frame-wall structures for seismic actions”, Ph.D. dissertation, University of Canterbury, 1985.

[4] T. Paulay y M. Priestley, "Stability of Ductile Structural walls," ACI Structural Journal, pp. 90, 385-392, 1993.
[5] Y. Chai y D. T. Elayer, "Lateral stability of reinforced concrete columns under axial reversed cyclic tension and compression,” ACI Structural Journal,, pp. 96(96), 780-789, 1999.

[6] Seismosoft, SeismoStruct Manual del Usuario, Pavia: Seismosoft Ldta., 2016.

[7] J. P. Almeida, D. Tarquini y K. Beyer, "Modelling Approaches for Inelastic Behaviour of RC Walls: Multi-level Assessment and Dependability of Results," Archives of Computational Methods in Engineering, vol. 23, n 1, p. 69-100., 2014.

[8] M. D’Aniello, F. Portioli y R. Landolfo, "Modelling issues of steel braces under extreme cyclic actions," Urban Habitat Constructions under Catastrophic Events, p. 1048, 2010.

[9] C. K. Herrick y M. J. Kowalsky, "Out-of-Plane Buckling Reinforced Structural Walls due to in Plane Loads," Journal of Structural Engineering, pp. 1-15, 2016.

[10] A. Rosso, "Out-of-plane instability of thin reinforce concrete walls under seismic loading”, Suiza: École Polytechnique Fédérale de Lausanne. Doctoral Thesis, 2018.

[11] A. Rosso, J. P. Almeida y K. Beyer, "Mechanical model to assess the vulnerability to out-of-plane instability of thin walls with a single layer of reinforcement," Bullettin of Earthquake Engineering, vol. 1, $n^{\circ} 10518,2018$. 